\title{
Criminologie
}

\section{La réforme du Code criminel canadien en matière de troubles mentaux et son impact sur la détention des justiciables}

\section{Danielle Laberge, Daphné Morin et Marie Robert}

Volume 28, numéro 2, 1995

La détention provisoire

URI : https://id.erudit.org/iderudit/017373ar

DOI : https://doi.org/10.7202/017373ar

Aller au sommaire du numéro

Éditeur(s)

Les Presses de l'Université de Montréal

ISSN

0316-0041 (imprimé)

1492-1367 (numérique)

Découvrir la revue

Citer cet article

Laberge, D., Morin, D. \& Robert, M. (1995). La réforme du Code criminel canadien en matière de troubles mentaux et son impact sur la détention des justiciables. Criminologie, 28(2), 61-83. https://doi.org/10.7202/017373ar
Résumé de l'article

This article discusses how pre-trial detention has become an important instrument in the treatment of the accused whose mental state has been questioned during the judicial process. This study is part of a major research trend centered on the hypothesis of criminalization of the mental illness. This hypothesis has been defined as a shift of groups of the population from the mental health system to the criminal justice system. First, the authors examine how the Criminal Code's dispositions and those regarding mental disorder, which have been revised in February 1992, can be associated with the Court's decisions regarding the release of the accused during the legal process. Then, the authors continue to look into the question concerning the articulation of dual decisional logic (judicial and psychiatric) by studying approximately 1000 cases heard before the Criminal and Penal Chamber of the Québec Court in Montréal in 1992-1993, in which the mental illness issue was raised. This analysis will try to demonstrate a link between pre-trial detention and mental health problems. It will also show that, despite the adoption of the principle of presumption against custody during assessment orders regarding mental disorder, the Court practices are changing slowly and the new dispositions are rarely used.
Tous droits réservés @ Les Presses de l'Université de Montréal, 1995
Ce document est protégé par la loi sur le droit d'auteur. L’utilisation des services d'Érudit (y compris la reproduction) est assujettie à sa politique d'utilisation que vous pouvez consulter en ligne.

https://apropos.erudit.org/fr/usagers/politique-dutilisation/ 


\section{LA REFFORME DU CODE CRIMINEL CANADIEN EN MATIÈRE DE TROUBLES MENTAUX ET SON IMPACT SUR LA DÉTENTION DES JUSTICIABLES ${ }^{1}$ \\ Danielle Laberge Daphné Morin Marie Robert ${ }^{2}$}

This article discusses how pre-trial detention has become an important instrument in the treatment of the accused whose mental state has been questioned during the judicial process. This study is part of a major research trend centered on the hypothesis of criminalization of the mental illness. This hypothesis has been defined as a shift of groups of the population from the mental health system to the criminal justice system. First, the authors examine how the Criminal Code's dispositions and those regarding mental disorder, which have been revised in February 1992, can be associated with the Court's decisions regarding the release of the accused during the legal process. Then, the authors continue to look into the question concerning the articulation of dual decisional logic (judicial and psychiatric) by studying approximately 1000 cases heard before the Criminal and Penal Chamber of the Québec Court in Montreal in 1992-1993, in which the mental illness issue was raised. This analysis will try to de. monstrate a link between pre-trial detention and mental health problems. It will also show that, despite the adoption of the principle of presumption against custody during assessment orders regarding mental disorder, the Court practices are changing slowly and the new dispositions are rarely used.

1. Les résultats présentés dans cet article ont été réalisés dans le cadre d'une recherche subventionnée par le Conseil québécois de la recherche sociale et par le ministère de la Sécurité publique du Québec.

2. Danielle Laberge, Ph. D., est professeure au département de sociologie de l'Université du Québec à Montréal. Elle est codirectrice du Groupe de recherche et d'analyse sur les politiques et les pratiques pénales (GRAPPP) est responsable du Collectif de recherche sur l'itinérance (CRI).

Daphné Morin, M. Sc. en sociologie, agit à titre d'agente de recherche et de pianification au département de sociologie de !'Université de Montréal.

Marie Robert, M. Sc. en sociologie, prépare actuellement un doctorat dans la même discipline au département de sociologie de l'Université du Québec à Montréal.

Toutes deux sont membres du Groupe de recherche et d'analyse sur les politiques et les pratiques pénales. 
L'hypothèse de la criminalisation des personnes atteintes de troubles mentaux est débattue depuis déjà quelques décennies. De façon générale, ce phénomène a le plus souvent été décrit comme le transfert, vers le système pénal, de groupes de la population auparavant pris en charge par le réseau des services de santé mentale. Malgré les efforts de la recherche, les analyses sur ce phénomène de criminalisation n'ont pas encore véritablement traité l'une des facettes fondamentales du processus qu'est le traitement réservé à ces affaires par le système judiciaire, parmi lesquelles on trouve l'usage fort répandu de la détention provisoire. Le présent article s'intéresse donc à la détention provisoire comme mesure importante de gestion du traitement judiciaire de prévenus dont l'état mental fait l'objet d'un questionnement au cours du processus. En d'autres termes, nous voulons saisir les modalités selon lesquelles le tribunal décide de détenir ou non un prévenu au cours du processus judiciaire lorsque son état mental a été mis en doute au début des procédures.

Dans un premier temps, nous examinerons la réforme du Code criminel canadien en regard des troubles mentaux, adoptée en février 1992, et qui a modifié le rôle de l'état mental dans le traitement judiciaire. Tout en présentant les grandes lignes de cette réforme, nous insisterons plus particulièrement sur les différentes procédures où la détention provisoire occupe ou est susceptible d'occuper une place importante. Dans un deuxième temps, nous procéderons à l'analyse d'une centaine de causes entendues devant la chambre criminelle et pénale de la cour du Québec à Montréal au cours de 1'année 1992-93 pour lesquelles la question des troubles mentaux a été soulevée. Cette analyse examinera de façon particulière l'usage de la détention provisoire. Il est certain qu'un tel examen ne constitue en aucune façon une évaluation de l'application des nouvelles dispositions du Code criminel, mais il devrait permettre d'illustrer la dynamique à l'œuvre et de suggérer des pistes pour de futures recherches.

\section{LE CADRE LÉGISLATIF : L'INTERFACE ENTRE LES LOGIQUES JUDICIAIRE ET PSYCHIATRIQUE}

Dans l'examen de la question de la criminalisation des troubles mentaux, l'intervention de la police, et particulièrement l'arrestation, est sans doute l'étape qui a été la plus fouillée (Teplin, 1983; Murphy, 1986; Davis, 1992; Schellenberg et al., 1992 : Laberge et Morin, 1993). À partir de ces études et d'autres plus générales portant sur la mise en forme pénale, deux constats sont essentiels. D'abord, pour qu'il y ait criminalisation, les policiers doivent nécessairement saisir un tribunal de l'affaire, i.e. prendre la décision de renvoyer l'affaire devant le système judiciaire plutôt que de régler la situation problématique d'une autre façon (le renvoi vers l'urgence psychiatrique ou le règlement de l'affaire de manière informelle, par 
exemple $^{3}$ ). Deuxièmement, dans le cas où ils décident de porter l'affaire devant le tribunal, la question de la mise en liberté du prévenu avant sa comparution devant le juge doit être tranchée. Dans ce processus décisionnel, que l'accusé ait ou non des problèmes d'ordre psychiatrique, que ces problèmes aient ou non été identifiés par les policiers, ces derniers ont à faire face aux mêmes décisions.

Au Canada, rien dans le Code criminel n'est prévu à cette étape des procédures pour introduire une autre logique décisionnelle qui ferait intervenir l'état mental d'un prévenu. En d'autres termes, ce sont les mêmes règles légales qui s'appliquent pour déterminer si l'affaire doit être portée en justice et, le cas échéant, si le prévenu doit faire l'objet d'une détention sous garde en attendant de comparaître devant un juge. Cela ne veut pas dire que l'état mental d'un suspect ne peut être invoqué pour justifier sa garde à vue, mais il doit alors être interprété à l'intérieur des catégories prévues par la loi, et qui sont identiques pour tous. Ainsi, les motifs que peut invoquer la police pour maintenir sous garde un suspect sont de deux ordres : 1) l'intérêt public ý compris la nécessité «i) d'identifier la personne; ii) de recueillir ou conserver une preuve de l'infraction ou une preuve qui y soit relative : iii) d'empêcher que l'infraction se poursuive ou se répète, ou qu'une autre infraction soit commise "; 2) des motifs raisonnables de croire que l'accusé ne se présentera pas au tribunal (C. cr. 497[1]). En principe, les prévenus atteints de troubles mentaux sont susceptibles d'être libérés en attendant leur comparution devant le tribunal par voie de sommation ou de citation à comparaître, au même titre que les autres prévenus.

Au tribunal, la situation n'est cependant pas la même. Deux logiques peuvent être invoquées et sont l'ceuvre dans la décision de détenir les justiciables en cours de procédure : la logique judiciaire et la logique psychiatrique. Ces dernières ne sont pas mutuellement exclusives, et le suspect dont l'état mental est mis en doute peut donc être détenu en regard de l'une ou de l'autre. Le tribunal peut faire appel à une logique à l'une des étapes des procédures, pour justifier par la suite la détention provisoire par les motifs propres à l'autre logique. La détention provisoire de justiciables atteints de troubles mentaux a donc ceci de particulier qu'elle repose sur l'articulation d'une double perspective décisionnelle encore mal saisie, et dont l'impact dans le traitement judiciaire de ces causes $n$ 'a pas encore fait l'objet d'études approfondies.

Lorsqu'une personne fait l'objet d'une poursuite judiciaire, la question de savoir si elle doit ou non être détenue pendant les procédures compte parmi les décisions les plus importantes dans le traitement de l'affaire parce

3. Légalement, toute personne peut faire une dénonciation devant un juge de paix (C. cr. 505); toutefois, dans la plupart des cas, c'est la situation que nous avons décrite qui prévaut. 
qu'elle risque d'influencer la suite des événements, depuis le plaidoyer et l'orientation d'une défense jusqu'à l'issue finale des procédures. De plus, sur le plan des droits fondamentaux, la détention préventive est décrite comme étant la violation la plus grave du droit à la liberté (Desrosiers, s. d.: 4). Dans la logique judiciaire, la question de la détention provisoire doit être déterminée à la lumière des principes de justice fondamentale énoncés dans la Charte canadienne des droits et libertés, en particulier ceux de la protection contre la détention ou l'emprisonnement arbitraire (art. 7), de la présomption d'innocence (art. 11d) et du droit «de ne pas être privé sans juste cause d'une mise en liberté assortie d'un cautionnement raisonnable » (art. 1 le).

Le tribunal peut faire appel à deux motifs pour justifier sa décision de détenir ou non un suspect pendant les procédures. Le motif principal se rapporte à la nécessité de détenir le prévenu pour s'assurer qu'il soit présent au tribunal pour être en mesure de le traiter selon la loi; le motif secondaire se réfère à la nécessité de la détention pour la protection et la sécurité du public (C. cr. 515[10]). Il faut ajouter que le second motif n'est examiné que si la cour est d'avis que l'accusé peut être libéré sans craindre qu'il ne tente d'échapper à la justice. En d'autres termes, le tribunal détermine si le suspect, considérant les garanties qu'il peut offrir pour rassurer la cour de sa présence au tribunal à sa demande et le danger présumé ou futur qu'il représente pour l'intérêt public ou la sécurité du public, a droit à une remise en liberté. Si cette décision est habituellement prise au début des procédures, soit à l'enquête sur la mise en liberté par voie judiciaire (mieux connue sous le nom d'enquête sur cautionnement), elle peut être révisée en cours de procédure.

Dans le cas d'une population atteinte de troubles mentaux, cette question se pose exactement dans les mêmes termes. Cependant, dans ces cas particuliers, d'autres circonstances peuvent alors intervenir, qui ramènent à nouveau la question de la détention provisoire: une logique psychiatrique se surajoute à la logique judiciaire dans le processus décisionnel. Les autres circonstances auxquelles nous faisons référence renvoient, pour l'essentiel, aux deux moments où l'état mental d'un justiciable peut intervenir dans le traitement judiciaire. Dans la réforme du Code criminel adoptée en 1992, le législateur a réaffirmé ces deux moments fondamentaux que sont: 1) le moment de l'infraction - l'accusé était-il atteint de troubles mentaux de façon à dégager sa responsabilité criminelle? 2) les procédures judiciaires - l'accusé est-il atteint de troubles mentaux qui le rendent inapte à subir son procès? Pour trancher ces questions de responsabilité criminelle et d'aptitude à subir le procès, la cour doit émettre des ordonnances d'évaluation de l'état mental de l'accusé. C'est dans le cadre de ces évaluations que la question de la mise en liberté de l'accusé se pose de nouveau.

Dans les nouvelles dispositions du Code criminel, le législateur énonce clairement le principe de la «priorité à la mise en liberté » qui doit guider la 
décision du tribunal (C. cr. 672.16[1]). En d'autres termes, les accusés soumis à ces ordonnances d'évaluation de leur état mental doivent être libérés pendant la durée des ordonnances, à moins que cette détention soit nécessaire. Or, les deux logiques peuvent ici intervenir pour justifier cette détention. D'une part, une logique psychiatrique qui s'articule autour de la nécessité de la détention pour évaluer l'état mental. Dans ce cas, deux critères sont énoncés : 1) la détention est nécessaire pour l'évaluation de l'état mental; 2) considérant le témoignage d'un médecin, la détention est souhaitable pour évaluer l'état mental de l'accusé et celui-ci y consent. D'autre part, une logique judiciaire dont les principes s'appliquent à l'ensemble de la population de justiciables et qui se rattache, comme nous l'avons vu précédemment, aux critères retenus pour déterminer si l'accusé peut ou non obtenir une mise en liberté provisoire.

\subsection{L'ordonnance d'évaluation}

La durée des ordonnances d'évaluation de l'état mental est au cœur même de la question de la détention provisoire dans le cadre de l'application de la seule logique psychiatrique. C'est d'ailleurs dans ce contexte que de nombreuses critiques ont été formulées à l'égard de l'ancienne disposition (Davis, 1994). En effet, faire l'objet d'une mesure aussi contraignante sur la seule base de son état mental devrait permettre de limiter au minimum la durée de la mesure. Dans la réforme adoptée, les énoncés traitant de la durée ont donc été modifiés, particulièrement ceux touchant à l'évaluation de l'aptitude à subir son procès, afin de réduire la durée des ordonnances.

Cependant, il est assez étonnant de constater que le législateur maintient la possibilité d'étendre sur une période aussi longue qu'auparavant, soit pendant 60 jours, la durêe des mêmes ordonnances. Ainsi, dans les cas les plus fréquents d'ordonnance d'évaluation de l'état mental pour déterminer l'aptitude de l'accusé à subir son procès, la durée de l'ordonnance a été limitée à cinq jours ouvrables desquels sont exclues les journées nécessaires au transport pour les déplacements entre la cour et le service d'expertise. Aussi restrictive soit-elle, cette limitation peut être facilement contournée grâce aux ouvertures laissées dans la loi. La première permet d'étendre sur une période de 30 jours la durée de l'ordonnance si le poursuivant et l'accusé y consentent; un énoncé plutôt étrange, considérant le fait que l'évaluation vise précisément à déterminer si l'accusé est dans un état d' « incapacité en raison de troubles mentaux d'assumer sa défense, ou de donner des instructions à un avocat... (C. cr. art. 2.) ${ }^{4}$ ». La deuxième

4. Dans la réforme du Code criminel de 1992, le législateur a circonscrit les champs d'application de l'incapacité : « $a$ ) comprendre la nature ou l'objet des poursuites; $b$ ) comprendre les conséquences éventuelles des poursuites; $c$ ) communiquer avec son avocat. » (C. cr. art. 2). 
permet au tribunal d'émettre une ordonnance d'une durée de 60 jours, si des circonstances exceptionnelles l'exigent. Dans ce dernier cas, la référence au consentement de l'accusé n'apparaît plus.

Il n'y a aucune obligation dans la loi actuelle de limiter à certains lieux particuliers, comme les hôpitaux par exemple, la responsabilité de faire les évaluations de l'état mental d'un prévenu. La seule restriction concerne les personnes autorisées à faire ces expertises : celles-ci doivent détenir le droit d'exercer la médecine dans la province. Cette imprécision laisse donc grande ouverte la possibilité de réaliser ces expertises en milieu carcéral, comme c'était le cas avant la réforme. Cela signifie que si l'accusé fait l'objet d'une mesure de détention pendant l'ordonnance d'évaluation de l'état mental, il est très probable qu'il sera détenu dans une institution carcérale.

Dans la réforme du Code criminel, l'ajout de certaines dispositions relatives à la question de l'aptitude à subir son procès donne la possibilité d'étendre l'usage de la détention provisoire à cette catégorie de justiciables. Ainsi, dans le cas où un justiciable est déclaré apte à comparaître après avoir fait l'objet d'une ordonnance d'évaluation, il pourra se voir imposer une nouvelle ordonnance de maintien en détention dans un hôpital si le tribunal a "des motifs raisonnables de croire qu'il deviendrait inapte s'il était remis en liberté » (C. cr. 672.29). Une limite est cependant imposée au tribunal. La disposition ne s'applique qu'aux individus qui étaient sous garde pendant que l'ordonnance d'évaluation de l'état mental était en vigueur.

Il s'agit bien d'un cas où les deux logiques sous-jacentes à la mise en détention provisoire peuvent se superposer ou encore où le psychiatrique s'affirme à la place du judiciaire. D'une part, la limite qui restreint l'application de la mesure aux seuls détenus pendant l'ordonnance ne spécifie pas si cette détention avait un caractère judiciaire ou psychiatrique. D'autre part, dans le cas où la détention pendant l'ordonnance d'évaluation reposait sur la seule logique psychiatrique (i.e. si la détention était jugée nécessaire pour évaluer l'état mental de l'accusé), cette seconde ordonnance s'y rattache (éviter que l'accusé ne redevienne inapte, s'il était remis en liberté). Il faut donc comprendre que le législateur autorise maintenant un tribunal à maintenir en détention provisoire un individu pendant toute la durée des procédures sur la seule base d'une perspective psychiatrique.

\subsection{Le verdict d'inaptitude}

En ce qui concerne le verdict d'inaptitude à subir son procès, nous voulons nous attarder sur quatre points particuliers qui concernent directement la détention provisoire : l'issue du verdict; le délai dans la décision à rendre; le traitement pour rendre apte; le délai pour une nouvelle comparution 
de l'accusé après un avis d'aptitude. La réforme a modifié de façon importante les issues possibles dans le cas des verdicts d'inaptitude ou de nonresponsabilité criminelle pour cause de troubles mentaux. Ainsi, lorsque le tribunal rend un verdict d'inaptitude à subir son procès, le tribunal ou la commission d'examen disposent de deux choix : 1) une libération conditionnelle ; 2) une détention à l'hôpital ${ }^{5}$. Pour déterminer son choix, le tribunal ou la commission d'examen doit rendre « la décision la moins sévère et la moins privative de liberté [...] compte tenu de la nécessité de protéger le public face aux personnes dangereuses, de l'état mental de l'accusé et de ses besoins, notamment de la nécessité de sa réinsertion sociale» (C. cr. 672.54). Il est donc clair que si l'on opte pour la détention à l'hôpital, le prévenu pourra être maintenu en détention provisoire jusqu'au moment où il sera jugé apte à subir son procès. Cette période de détention sera dorénavant limitée dans le temps et fixée, selon la nature de l'infraction, à une période maximale ${ }^{6}$. De plus, il faut noter que le principe retenu étend, bien au-delà de ce qu'autorise le droit civil, les pouvoirs de détention de l'État pour des motifs controversés (prédiction de dangerosité), vagues (état mental, besoins), ou très globaux (réinsertion sociale).

Lorsque la cour rend un verdict d'inaptitude ou de non-responsabilité criminelle pour cause de troubles mentaux, deux instances possèdent le pouvoir de trancher la question de l'issue : le tribunal ou la commission d'examen. La décision qui doit être rendue peut être différée jusqu'à une période de 90 jours. C'est donc dire qu'une fois encore, pendant cette période d'attente de décision, l'accusé peut être maintenu en détention provisoire.

Suite à un verdict d'inaptitude à subir son procès, le tribunal peut ordonner le traitement des accusés déclarés inaptes, pour une période n'excédant pas 60 jours, si le tribunal est convaincu, sur la foi du témoignage d'un médecin (critère et preuve nécessaire C. cr. 672.59[1][2]), que cette personne redeviendrait apte dans ce délai si un traitement lui était administré. Une restriction est cependant imposée. L'ordonnance doit être émise avant que le tribunal ou la commission ait rendu sa décision quant à l'issue la plus appropriée. Cela signifie que pendant la période de validité de cette ordonnance, si le prévenu est détenu, il le sera en conformité avec une ordonnance qui précède le verdict d'inaptitude. On peut alors penser que cette détention relèverait alors de l'une ou de l'autre logique.

La commission d'examen, chargée de rendre les décisions ou de les réviser, doit ordonner le renvoi du prévenu devant le tribunal si elle est

5. Dans le cas du verdict de non-responsabilité criminelle pour cause de troubles mentaux, la libération inconditionnelle s'offre comme une troisième option possible (C. cr. 672[54a]).

6. Il s'agit là du principe du capping non encore en vigueur. 
d'avis qu'il est en mesure de subir son procès. En attendant sa recomparution devant le tribunal, la commission peut ordonner que le prévenu soit détenu à l'hôpital si elle a «des motifs raisonnables de croire que l'accusé deviendra inapte à subir son procès s'il est mis en liberté" (C. cr. $672.49[1])$.

\section{UN EXAMEN EMPIRIQUE : TROUBLES MENTAUX ET DÉTENTION PROVISOIRE}

Depuis déjà plusieurs années à la chambre criminelle et pénale de la cour du Québec à Montréal, le programme «médecin à la cour " se propose d'évaluer sommairement des justiciables présentant potentiellement des problèmes de santé mentale. Pour l'essentiel, cette évaluation répond à des recommandations des policiers qui ont constaté des problèmes de dysfonctionnement ou de dangerosité potentielle lors de l'arrestation des justiciables en question. Il arrive aussi, quoique beaucoup plus rarement, que cette évaluation se produise à la demande du tribunal suite à une première comparution. Le médecin qui réalise cet examen recommande au tribunal d'approfondir l'évaluation de l'aptitude du justiciable ou encore considère que la personne est apte à comparaître. Notons immédiatement que cette recommandation du médecin est presque toujours suivie par le tribunal.

L'analyse empirique que nous présentons ici se fonde sur l'étude de 117 causes ayant fait l'objet d'une évaluation dans le cadre du programme «médecin à la cour ». Un tel choix se justifie de diverses façons. En premier lieu, notons que les policiers jouent un rôle absolument capital dans l'identification des personnes souffrant potentiellement de problèmes de santé mentale. De nombreuses études aussi bien canadiennes qu'américaines (Arboleda-Florez et Holley, 1988: Holley et Arboleda-Florez, 1988; Laberge et al., à paraître: Teplin, 1984a, b; Teplin et Pruett, 1992) ont permis de constater que les policiers sont des informateurs privilégiés dans le domaine de la santé mentale et de son traitement judiciaire. Cela n'est pas surprenant, compte tenu du fait que ce sont les acteurs pénaux qui ont les contacts les plus directs avec les justiciables, intervenant "sur le vif » en quelque sorte. Les études ont aussi permis de constater la très grande importance accordée à leurs demandes d'examen.

Le choix du programme «médecin à la cour» nous permet donc de viser, dès son entrée dans le système pénal, la clientèle susceptible de faire l'objet de mesures à caractère psychiatrique. Le choix se fonde également sur des raisons pratiques : en effet, la défense de non-responsabilité criminelle pour cause de troubles mentaux, qui constitue l'autre mesure importante dans ce domaine, est à la fois rarement utilisée et très difficile à repérer dans l'ensemble des causes entendues devant le tribunal. L'échantillon constitué sur la base de ce programme nous permet donc de faire une 
sélection la plus large des cas possibles. Cependant, il n'est pas sans faille, puisqu'il ne nous garantit pas que certains cas n'émergeront pas au cours du processus, mais ces derniers ne sont pas suffisamment nombreux pour invalider une première démarche exploratoire.

Nous avons done constitué, à partir de la banque de données du service de probation, qui assure la gestion du programme «médecin à la cour», un échantillon de 117 causes sur la base suivante : nous avons retenu toutes les causes impliquant une femme inculpée $(n=21)$ et nous avons sélectionné 96 causes où des hommes se trouvaient dans la même situation; pour ces derniers, nous avons sélectionné au hasard 48 causes pour lesquelles il n'y a pas eu de recommandation d'évaluation approfondie de l'aptitude, et 48 causes pour lesquelles cette recommandation était faite. Le lecteur devra donc garder deux éléments présents à l'esprit : dans cet échantillon, le rapport entre les hommes et les femmes est biaisé par une surreprésentation des femmes par rapport aux hommes. Un tel choix, relativement courant dans les procédures de recherche, permet de disposer d'un nombre suffisant de causes pour être en mesure de tenir compte de la dimension sexuelle, ce qu'un échantillon arbitraire ne permettrait pas. D'autre part, afin de permettre la comparaison, nous avons équilibré la composition de l'échantillon des hommes.

Nous présenterons quelques caractéristiques permettant de tracer un profil de l'échantillon ainsi constitué. Les justiciables ont entre 18 et 79 ans, la moyenne d'âge se situe à 36,5 ans. Une proportion importante est célibataire $(68,4 \%)$. Plus de la moitié vit de fonds gouvernementaux $(66,7 \%)$ alors que seulement $13,3 \%$ déclarent un revenu d'emploi, et $12,4 \%$ n'auraient aucune source de revenu. Plus de la moitié de l'échantillon a une histoire psychiatrique connue $(55,6 \%), 31,6 \%$ n'ont pas d'antécédents de cette nature, alors que nous ne disposons pas de l'information pour $12,8 \%$ des justiciables. Quant aux antécédents judiciaires, ils présentent un profil similaire : $56,7 \%$ des justiciables en ont, alors que $32,5 \%$ n'en ont pas, et que pour les derniers $11,1 \%$ nous ne disposons d'aucune information.

L'ensemble de nos justiciables ont été mis sous garde par les policiers qui ont, de plus, suggéré qu'ils soient examinés par le médecin relativement à leur état mental. Lors de la première comparution, un peu plus du tiers du groupe est libéré, alors que tous les autres justiciables vont être détenus. Les études portant sur la détention provisoire ont montré toute la difficulté d'identifier un nombre restreint d'indicateurs qui permettraient de prédire assez correctement les décisions du tribunal à ce chapitre (Laberge et Landreville, 1993). Ces indicateurs tentent généralement d'opérationnaliser les critères légaux qui constituent le cadre décisionnel à l'intérieur duquel fonctionnent les tribunaux. S'ils peuvent être variables d'une juridiction à 
l'autre - et d'une étude à l'autre - ils renvoient pour l'essentiel à deux grandes catégories de préoccupations : l'assurance que la personne accusée sera présente au tribunal pour y être jugée, et le danger potentiel qu'elle représente pour les autres.

Le premier de ces grands critères est généralement appréhendé par le biais des variables servant à cerner la stabilité individuelle. Selon cette logique, les gens ont d'autant plus à perdre en cas de non-comparution qu'ils mènent une vie stable. Mais on peut mettre en doute le caractère réversible de cette proposition : ce n'est pas parce que les gens ont moins à perdre qu'ils ne vont pas se présenter. C'est donc dire que si une situation plus privilégiée permet de laisser augurer une certaine tolérance de la part du tribunal, il est plus difficile de prédire l'issue dans les situations où les personnes sont désavantagées. C'est ainsi que, dans notre échantillon, on ne note entre les personnes détenues et les personnes libérées aucune différence significative quant au sexe, au statut civil, ou au mode d'habitation. Par contre, on note une légère différence en ce qui concerne les sources de revenus : les personnes ayant des revenus d'emploi sont plus souvent libérées, alors que les personnes recevant l'aide sociale sont légèrement plus souvent détenues; cette différence est néanmoins ténue. C'est dans les situations où cette problématique de l'instabilité est très marquée que le lien apparaît évident. Ainsi, les personnes considérées comme itinérantes sont peu nombreuses dans notre échantillon $(\mathrm{n}=15)$, mais elles sont toutes détenues à une exception près.

La seconde dimension susceptible d'entrer en ligne de compte dans la décision de détenir est relative à la dangerosité potentielle des contrevenants. Bien entendu, cette question est extrêmement complexe et son opérationnalisation n'a pas manqué de soulever de nombreux débats (Landreville, 1993). En effet, la dangerosité se limite-t-elle à des comportements potentiels susceptibles de mettre la vie ou la santé des autres en danger? Doit-on inclure dans cette évaluation la probabilité de recommettre un délit? un délit de même nature? Le délit que fait l'objet de la présente évaluation ${ }^{7}$ constitue-t-il en lui-même un indicateur devant être retenu?

De toute évidence, nous ne trancherons pas le débat dans le cadre de cet article. Il nous semblait néanmoins important d'examiner quelques-unes des caractéristiques souvent proposées à titre de critères de décision. Nous examinerons donc la nature du délit, la présence d'antécédents judiciaires et la perception selon laquelle la personne constitue un danger pour les autres ou pour elle-même. Pour la présente analyse, nous avons catégorisé sous quatre rubriques l'ensemble des infractions dont étaient accusés les justiciables

7. Bien entendu, nous ne parlons pas ici des délits pour lesquels il existe, dans le Code criminel, une présomption de détention et où le fardeau de la preuve repose sur l'accusé. 
de l'échantillon. Nous avons différencié ici les crimes graves contre la personne des autres crimes contre la personne ${ }^{8}$, en partant de l'hypothèse qu'il pouvait s'agir là d'une distinction que le tribunal serait susceptible de mettre en œuvre.

Tableau 1

Décision quant à la libération selon la nature de l'accusation

\begin{tabular}{|l|c|c|c|c|c|}
\hline \multirow{2}{*}{ STATUT } & \multicolumn{5}{|c|}{ ACCUSATION } \\
\cline { 2 - 6 } & $\begin{array}{c}\text { Contre la } \\
\text { personne } \\
\text { grave }\end{array}$ & $\begin{array}{c}\text { Contre la } \\
\text { personne } \\
\text { autre }\end{array}$ & $\begin{array}{c}\text { Contre les } \\
\text { biens }\end{array}$ & $\begin{array}{c}\text { Autres } \\
\text { délits }\end{array}$ & Total \\
\hline \multirow{3}{*}{ Libéré } & 9 & 22 & 6 & 2 & 39 \\
& 23,1 & 56,4 & 15,4 & 5,2 & \\
& 31,0 & 53,7 & 22,2 & 20,0 & 36,4 \\
\hline \multirow{3}{*}{ Détenu } & 20 & 19 & 21 & 8 & 68 \\
& 29,4 & 27,9 & 30,9 & 11,8 & 63,6 \\
\hline Total & 69,0 & 46,3 & 77,8 & 80,0 & 107 \\
\hline
\end{tabular}

Données manquantes $=10$

Il existe effectivement une différence significative dans la nature des accusations entre les deux groupes de justiciables. Comme on pouvait s'y attendre, les personnes accusées d'infractions graves contre la personne ont de plus fortes chances d'être détenues, ce rapport s'inversant lorsqu'il s'agit d'autres crimes contre la personne. Ce qui ne manque pas d'étonner en revanche, c'est la très forte proportion de personnes accusées d'infractions contre les biens et qui seront détenues. Compte tenu des caractéristiques de notre population, nous pouvons supposer qu'une autre logique est ici à l'œuvre. La présence d'antécédents judiciaires, quant à elle, ne permet pas d'expliquer cette décision: près des deux tiers de l'échantillon $(63,5 \%)$ possèdent de tels antécédents et se retrouvent d'ailleurs dans des proportions similaires dans les deux groupes. Enfin, nous désirons voir dans quelle mesure la perception de la dangerosité est susceptible d'entraîner la détention. Les policiers, lors de l'arrestation, mentionnent, lorsqu'ils le croient pertinent, une dangerosité soit pour les autres, soit pour la personne elle-même. La mention de dangerosité pour les autres semble effectivement influencer la décision du tribunal.

8. Dans la catégorie "autres crimes contre la personne", nous retrouvons : voies de fait simples, voies de fait sur policier, agression sexuelle simple, menaces, extorsion, séquestration. 
Tableau 2

Décision quant à la libération selon la perception de dangerosité

\begin{tabular}{|l|l|l|c|}
\hline \multirow{2}{*}{ STATUT } & \multicolumn{3}{|c|}{ DANGEROSITÉ PERÇUE } \\
\cline { 2 - 4 } & Oui & Non & Total \\
\hline \multirow{3}{*}{ Libéré } & 15 & 22 & 37 \\
& 40,5 & 59,5 & 46,3 \\
& 31,3 & 68,8 & 43 \\
\multirow{3}{*}{ Détenu } & 33 & 10 & 53,8 \\
& 76,7 & 23,3 & 80 \\
\hline \multirow{2}{*}{ Total } & 68,8 & 31,3 & 100,0 \\
\hline
\end{tabular}

Chi carré $=10,86109$, n.s. $=, 00098$

Données manquantes $=37$

Les justiciabłes ont plus de chances d'être détenus lorsqu'ils sont perçus comme présentant un danger. Dans le cas d'une information allant dans ce sens, le lien semble donc probant. Néanmoins, cette information n'a pas été inscrite pour le tiers de notre échantillon; ce manque d'information concerne directement les personnes détenues. La seule perception de la dangerosité est donc insuffisante pour expliquer la mise en détention. Quant au danger pour soi-même, il est si rarement perçu par les policiers qu'il ne peut constituer un critère déterminant dans la décision du tribunal.

Nous avons aussi porté notre attention sur le contexte entourant l'intervention pénale. Bien que nous disposions de peu d'éléments pour le faire, une dimension est apparue très importante, celle de l'identité du plaignant d'origine. Qui porte plainte? Qui met en branle l'action judiciaire? Nous avons retenu cinq catégories de plaignants : l'entourage immédiat du justiciable (famille, conjoint, ami...); les citoyens sans aucun lien (propriétaire, gérant d'un commerce, témoin...); des membres du réseau socio-sanitaire (il s'agit surtout des hôpitaux); des membres d'entreprises publiques (école, banque, services municipaux); les policiers eux-mêmes. Comme on peut le constater à la lecture du tableau suivant, la décision du tribunal est très différente selon qu'il s'agit d'une demande provenant de l'entourage immédiat ou d'une autre source. 
Tableau 3

Décision quant à la libération selon l'identité du plaignant

\begin{tabular}{|l|c|c|c|c|}
\hline \multirow{2}{*}{ ST ATUT } & \multicolumn{4}{|c|}{ IDENTITÉ DU PLAIGNANT } \\
\cline { 2 - 5 } & $\begin{array}{c}\text { Réseau } \\
\text { privé }\end{array}$ & Citoyens & $\begin{array}{c}\text { Sphère } \\
\text { publique }\end{array}$ & Total \\
\hline \multirow{3}{*}{ Libéré } & 24 & 7 & 7 & 38 \\
& 63,2 & 18,4 & 18,5 & 36,2 \\
\hline \multirow{3}{*}{ Détenu } & 58,5 & 21,2 & 22,5 & 67 \\
& 17 & 26 & 24 & 63,8 \\
\hline \multirow{2}{*}{ Total } & 25,4 & 38,8 & 35,8 & 105 \\
\hline
\end{tabular}

Données manquantes $=12$

On peut ici s'interroger sur le sens variable que peut prendre, pour différentes catégories d'acteurs sociaux, le recours à l'appareil judiciaire. En effet, la nature des rapports avec le justiciable est susceptible d'expliquer des attentes très différentes de la part du renvoyant, en activant une affaire pénale. Des travaux portant sur différentes questions ont permis d'illustrer comment le fait de porter plainte représente souvent, dans l'esprit du renvoyant, une stratégie pour faire cesser un comportement, pour contrôler temporairement une personne, pour tenter de faire pression afin qu'elle s'amende. De telles attentes s'expliquent dans la perspective où l'on souhaite maintenir des liens, rendre la vie plus facile, plus harmonieuse. Il est évident que ces motivations n'animent pas certaines catégories de plaignants. Par ailleurs, les plaintes provenant de la famille assurent, du point de vue du tribunal, la présence d'un répondant potentiel; une telle situation peut parfois constituer une assurance quant à la présence future de l'accusé au tribunal.

En fait, la décision de libérer ou non est associée ici de façon très marquée à la problématique de la maladie mentale. Cette assertion s'appuie sur trois éléments : la présence d'antécédents psychiatriques; la perception du besoin d'un traitement psychiatrique; Ja décision de soumettre le justiciable à un examen approfondi pour évaluer son aptitude à comparaître. Cette dernière décision du tribunal permet d'ailleurs d'éclairer l'importance des deux autres questions. 
Tableau 4

Décision quant à la libération selon la décision d'envoyer ou non subir un examen approfondi

\begin{tabular}{|l|c|c|c|}
\hline \multirow{2}{*}{ STATUT } & \multicolumn{3}{|c|}{ EXAMEN APPROFONDI } \\
\cline { 2 - 4 } & Oui & Non & Total \\
\hline \multirow{3}{*}{ Libéré } & & 39 & 39 \\
& & 100,0 & 36,4 \\
\hline \multirow{3}{*}{ Détenu } & & 81,3 & 68 \\
& 59 & 9 & 63,6 \\
\hline \multirow{2}{*}{ Total } & 86,8 & 13,2 & 107 \\
\hline
\end{tabular}

Chi carré $=75,43107$ n.s. $=, 00000$

Données manquantes $=10$

Le renvoi pour examen de l'aptitude constitue un remarquable facteur de prédiction de la décision du tribunal quant à détenir ou à libérer. D'abord, notons qu'aucun examen n'a été demandé par le tribunal pour des personnes qui auraient été remises en liberté. Cette possibilité était déjà présente dans l'ancienne version des dispositions du Code criminel $^{9}$, et elle a été définie comme une option prioritaire lors des modifications apportées avec l'adoption du projet de loi C-30 (C. cr. 672.16). Aucun indice ne nous permet de comprendre un tel choix de la part du tribunal. Il est évident que la détention permet de procéder de façon commode aux examens, les personnes étant toujours disponibles dans ce contexte. Il est aussi possible que les critères à l'œuvre dans la décision de libérer soient fortement associés à la "normalité " des conditions de vie; il y aurait alors un recouvrement entre les critères favorisant la libération et ceux permettant de croire à un fonctionnement personnel satisfaisant ct suffisant pour procéder à l'audition de la cause. Nous examinerons d'ailleurs cette question plus longuement. Contrairement au rapport existant entre libération et absence d'examen approfondi, le lien entre détention et examen est moins évident mais il est néanmoins très marqué : on constate que neuf personnes sont détenues sans faire l'objet d'un examen, alors que 59 feront l'objet d'un tel examen.

Contrairement à ce que nous avions constaté pour les antécédents judiciaires, les antécédents psychiatriques ${ }^{10}$ discriminent, sur le plan statistique,

9. C. cr. $615.2 \mathrm{c}$.

10. Définis ici comme le fait d'avoir déjà eu des interventions à caractère psychiatrique : suivi exteme, médication, hospitalisation. 
entre personnes libérées et détenues. En effet, parmi les 59 personnes dont les antécédents psychiatriques sont connus, 42 seront détenues et 17 libérées. La perception du besoin de soins psychiatriques, indicateur qui prend un caractère immédiat du point de vue du tribunal, permet aussi de trancher entre les deux groupes, comme en témoigne le tableau 5. Cette information est fournie par le médecin qui procède à l'examen sommaire des justiciables.

Tableau 5

Décision quant à la libération selon ta perception de besoin de soins psychiatriques par le médecin de la cour

\begin{tabular}{|l|c|c|c|}
\hline \multirow{2}{*}{ STATUT } & \multicolumn{3}{|c|}{ BESOIN DE SOINS } \\
\cline { 2 - 4 } & Oui & Non & Total \\
\hline \multirow{3}{*}{ Libéré } & 5 & 32 & 37 \\
& 13,5 & 86,5 & 36,3 \\
\hline \multirow{3}{*}{ Détenu } & 8,9 & 69,6 & 65 \\
& 51 & 14 & 63,7 \\
\hline \multirow{2}{*}{ Total } & 78,5 & 21,5 & 102 \\
& 91,1 & 30,4 & 100,0 \\
\hline
\end{tabular}

Chi carré $=40,16995$ n.s. $=, 00000$

Données manquantes $=15$

Le besoin de traitement, fortement associé à la détention, semble aussi constituer un critère important dans la décision du médecin de recommander un examen approfondi" ${ }^{1}$. Parmi les 56 justiciables pour lesquels une telle recommandation a été faite, 48 auront été envoyés consulter pour examen approfondi, trois étant détenus sans pour autant faire l'objet d'une telle procédure, Sur la base de nos données, la thèse de la "criminalisation de la maladie mentale » semble confirmée à certains égards. Cette confirmation tient dans l'association quasi parfaite entre la détention et le besoin d'évaluation en profondeur. Parmi le groupe particulier que nous avons sélectionné, seule une très faible proportion de personnes sont détenues sans faire l'objet d'un examen approfondi. C'est dans cette perspective que l'on peut peut-être comprendre le fait qu'un nombre aussi important de personnes accusées de délits contre les biens se trouvent détenues, puisque c'est la nécessité d'un examen psychiatrique qui joue ici.

Avant de procéder à l'examen de l'issue des procédures judiciaires, nous allons nous pencher sur la question de la durée de la période d'observation. La période d'évaluation s'est étendue de 11 à 152 jours pour les

11. Une telle recommandation est à toutes fins utiles toujours suivie par le tribunal. 
justiciables de notre échantillon, la moyenne se situant à 19,5 jours. Rappelons que les modifications au Code criminel réduisaient la durée prescrite pour l'observation de 30 à 5 jours. Ces modifications ne semblent pourtant pas avoir affecté les pratiques concrètes dans ce domaine ${ }^{12}$. En effet, la nouvelle loi prévoit une première période d'évaluation de cinq jours ${ }^{13}$, et aucun justiciable de notre échantillon n'a été évalué dans un laps de temps aussi court.

\section{Tableau 6}

Durée de la période d'observation selon les nouveaux critères prévus au Code criminel canadien

\begin{tabular}{|c|c|}
\hline $\begin{array}{l}\text { Première évaluation } \\
\text { ( } 5 \text { jours) }\end{array}$ & $0.0 \%$ \\
\hline $\begin{array}{l}\text { Première prolongation } \\
\text { ( } 30 \text { jours) }\end{array}$ & $60,0 \%$ \\
\hline $\begin{array}{l}\text { Prolongation maximale } \\
\text { ( } 60 \text { jours) }\end{array}$ & $28,9 \%$ \\
\hline $\begin{array}{l}\text { Hors délai } \\
\text { (plus de } 65 \text { jours) }\end{array}$ & $10,2 \%$ \\
\hline
\end{tabular}

Sous l'ancienne loi, la durée d'observation maximale était de 60 jours $^{14}$, et la pratique voulait qu'on accorde deux semaines pour procéder à l'évaluation. L'examen de la répartition des données montre donc que les parties en cause semblent avoir maintenu leurs anciennes habitudes au lieu d'avoir recours à de nouvelles possibilités, notamment celle d'émettre une ordonnance de traitement pour rendre apte (C. cr. 672.54). Cette dernière comporte certaines contraintes que le tribunal préfère sans doute contourner.

Quant au lieu d'observation, les analyses montrent bien qu'en l'absence de limites claires imposées par le législateur, la prison demeure un lieu habituel pour procéder aux expertises psychiatriques demandées par le tribunal.

12. Des analyses portant sur une autre sous-population issue de ce programme " médecin à la cour " et constituée sur la base de l'apparition du justiciable à au moins deux examens sommaires dans deux causes différentes entre 1989 et 1994, tendent à confirmer ces premières constatations. En effet, à partir de ces données, nous avons constaté que la durée moyenne des ordonnances d'évaluation de l'aptitude avant mars 1992 était de 20,3 jours; la plus courte étant de 13 jours et la plus longue de 48 jours. À compter de mars 1992, on remarque même une augmentation. La durée moyenne atteint 25,2 jours, la plus courte période étant de 13 jours et la plus longue de 64 jours. Nous pouvons également ajouter que les analyses portant sur la première année d'entrée en vigueur de la réforme en Alberta vont tout à fait dans la même direction (Calgary WHO Centre for Research and Training in Mental Health, 1993).

13. Excluant les jours fériés et les jours de transfert d'une institution à l'autre.

14. C. cr. $615.3 \mathrm{~b}$. 
Dans le cas de notre échantillon, deux hypothèses sont principalement favorisées: 27 justiciables seront évalués à l'Institut Philippe Pinel de Montréal, 24 seront gardés dans une institution carcérale ${ }^{15}$ et examinés sur place par un psychiatre, et seulement 5 seront envoyés dans un hôpital pour y subir leur examen 16 .

$\mathrm{Au}$ retour de l'examen, 47 justiciables étaient jugés aptes à comparaître, alors que 6 étaient considérés inaptes. Ces 47 justiciables vont donc comparaître de nouveau devant le tribunal et, à cette étape des procédures, nous voyons $s$ 'inverser les rapports entre justiciables détenus et justiciables libérés. Nous retrouvons donc $30 \%$ des justiciables qui seront détenus jusqu'à la fin des procédures, alors que $70 \%$ sont libérés immédiatement après la première comparution, ou suite au retour devant le tribunal après une évaluation positive quant à leur aptitude à comparaître. Pourtant, cette libération ne garantit en rien qu'elle se maintiendra jusqu'à la fin des procédures. L'examen approfondi d'un certain nombre de dossiers nous a permis de constater que des justiciables pouvaient être réincarcérés dans le courant des procédures, et ce, pour différents motifs : non-comparution subséquente, rupture des conditions de remise en liberté, mise en détention dans le cadre d'une autre poursuite en cours ou d'une nouvelle poursuite. Le portrait à cet égard est d'une telle complexité qu'il est impossible d'en rendre compte sur le plan statistique. Nous retenons deux choses de cette situation: premièrement, le tribunal a pris la décision de libérer le justiciable; deuxièmement, cela n'implique pas que tous les justiciables libérés soient demeurés libres par la suite.

La décision de libérer n'implique pas pour autant que le tribunal perde complètement de vue les problèmes potentiels de santé mentale des justiciables. Bien que nous n'ayons vu aucun cas où le tribunal utilise la nouvelle disposition du code permettant le maintien en détention dans un hôpital (C. cr. 672.29) afin de prévenir l'inaptitude du justiciable, d'autres mesures sont mises en œuvre, qui signalent clairement des préoccupations à cet égard. Ces mesures sont la plupart du temps suggérées par le médecin lors de l'examen sommaire, ou par le psychiatre dans le cadre de son rapport d'examen approfondi.

15. Centre de prévention de Montréal (Parthenais) et Maison Tanguay.

16. Nous ne disposons d'aucune information pour 3 cas sur 59. 
Tableau 7

Présence des mesures à caractère psychiatrique ou psychosocial à la libération selon la présence ou non d'un examen approfondi

\begin{tabular}{|l|c|c|c|}
\hline \multirow{2}{*}{$\begin{array}{c}\text { MESURES } \\
\text { PSYCHIATRIQUES }\end{array}$} & \multicolumn{3}{|c|}{ PRÉSENCE D'EXAMEN } \\
\cline { 2 - 4 } & $\begin{array}{c}\text { Libéré sans } \\
\text { examen }\end{array}$ & $\begin{array}{c}\text { Libéré après } \\
\text { examen }\end{array}$ & Total \\
\hline \multirow{3}{*}{ Oui } & 19 & 28 & 47 \\
& 40,4 & 59,6 & 61,8 \\
Non & 48,7 & 75,7 & 29 \\
& 20 & 9 & 98,2 \\
\hline \multirow{2}{*}{ Total } & 69,0 & 31,0 & 76 \\
\hline
\end{tabular}

On constate, d'après l'examen des données, que ces recommandations sont présentes pour une majorité d'accusés, qu'ils aient été ou non soumis à un examen approfondi de leur aptitude à comparâ̂tre. Néanmoins, les personnes ayant été envoyées consulter pour une évaluation en profondeur font presque systématiquement l'objet de telles mesures. C'est donc dire que les experts ne limitent pas leur travail à la simple question de l'aptitude, mais fournissent au tribunal des pistes possibles d'intervention. Comme l'a souligné Davis (1994), ils maintiennent ainsi leurs anciennes pratiques. Leurs recommandations sont prises au sérieux par le tribunal qui y répond de façon systématique.

De nombreux travaux de recherche portant sur la détention provisoire ont abondamment étudié l'impact potentiel de cette dernière sur la décision de culpabilité de l'accusé d'une part, et la sévérité de la sentence, d'autre part. Nous examinerons donc ici l'issue de la procédure en comparant non pas deux mais trois groupes; les justiciables libérés lors de la première comparution, les justiciables libérés après avoir subi un examen approfondi pour déterminer leur capacité à subir leur procès, et les justiciables détenus tout au long des procédures, qu'ils aient subi ou non cet examen. Le tableau 8 présente cette issue en trois grandes catégories: les justiciables reconnus coupables, les justiciables libérés ${ }^{17}$ et ceux placés sous les bons soins du lieutenant gouverneur.

17. Cette catégorie inclut les acquittements, les retraits de plainte, les libérations inconditionnelles et les personnes pour lesquelles la seule mesure a été une ordonnance 810 (garder la paix). 


\section{Tableau 8}

Nature de la décision quant à l'issue des procédures

selon le statut lors des procédures

\begin{tabular}{|l|c|c|c|c|}
\hline \multirow{2}{*}{$\begin{array}{c}\text { DÉCISION- } \\
\text { ISSUE }\end{array}$} & \multicolumn{4}{|c|}{ STATUT } \\
\cline { 2 - 5 } & Libéré & $\begin{array}{c}\text { Libéré après } \\
\text { examen }\end{array}$ & Détenu & Total \\
\hline \multirow{3}{*}{ Coupable } & 25 & 20 & 16 & 61 \\
& 64,1 & 57,1 & 51,6 & 58,1 \\
& 41,0 & 32,8 & 26,2 & 31 \\
\hline \multirow{3}{*}{ Libéré } & 14 & 14 & 3 & 29,5 \\
& 23,1 & 40,7 & 9,6 & 13 \\
\hline \multirow{3}{*}{ OLG } & 45,2 & 45,2 & 9,6 & 12,4 \\
& & 1 & 12 & 105 \\
\hline \multirow{2}{*}{ Total } & 39 & 2,9 & 38,7 & 100,0 \\
\hline
\end{tabular}

Données manquantes $=12$

Comme on peut le constater, deux lignes de démarcation apparaissent : d'abord, les mesures de libération se retrouvent essentiellement dans les deux catégories de justiciables libérés en cours de procédure, qu'ils aient ou non été renvoyés pour examen approfondi de leur aptitude à comparaître. Le fait d'être détenu ne semble pas favoriser ce genre d'issue. Le second aspect qu'il est important de souligner concerne les ordonnances du lieutenant gouverneur (OLG) dans le cadre d'une défense de non-responsabilité. Cette dernière est utilisée et retenue par le tribunal à peu près exclusivement lorsque les personnes sont détenues tout au long des procédures, et ce, bien qu'un nombre important de justiciables, libérés par la suite, aient fait l'objet d'un examen approfondi quant à leur aptitude. Le maintien en détention de ces personnes est peut-être justifié par des éléments signalant des difficultés importantes de fonctionnement et les risques éventuels, pour elles-mêmes ou pour les autres, de leur remise en liberté.

La nature de la sentence constitue un deuxième élément important pour évaluer le lien potentiel entre détention en cours de procédure et issue pénale. Le tableau suivant, distinguant les personnes détenues et celles libérées, montre une différence notable dans la nature de la sentence imposée par le tribunal. En effet, les personnes détenues ont de très fortes chances d'être condamnées à une peine de détention, alors que les personnes libérées en cours de procédure sont pour la plupart condamnées à une sentence suspendue avec probation. 
Il n'est évidemment pas possible, comme c'est d'ailleurs le cas dans la vaste majorité des études empiriques, de déterminer si la détention accroît les chances d'être jugé coupable, en premier lieu, et d'être condamné à une peine plus sévère, en second lieu. Les tenants d'une telle hypothèse mettent de l'avant les effets négatifs de la détention sur la capacité du justiciable à assurer sa défense, sur l'image négative qu'une telle situation crée dans l'esprit du tribunal, ainsi que sur l'incapacité dans laquelle il se trouve de faire la démonstration que sa conduite est maintenant irréprochable (ce que peuvent faire valoir les personnes remises en liberté). L'hypothèse concurrente suppose plutôt le recouvrement des critères à l'œuvre dans l'une et l'autre décision. En d'autres termes, les motifs qui incitent le tribunal à détenir les personnes pendant les procédures seraient les mêmes que ceux qui justifieraient une peine de détention. En ce qui concerne nos données, les premières analyses ne permettent pas d'identifier des caractéristiques marquées entre chacune des trois catégories, et il nous semble que des dimensions situationnelles lors de la comparution même, et pour lesquelles nous ne disposons pas d'informations, sont susceptibles de jouer.

S'il est évident que la décision de la détention au cours des procédures est liée à l'issue des procédures d'une part, et de la sentence d'autre part, qu'en est-il des dimensions psychiatriques de l'affaire? Comme nous l'avons souligné, les personnes détenues pendant les procédures voient leur affaire prendre deux directions principales : une direction pénale (peine d'incarcération) et une direction psychiatrique (OLG). Le tableau suivant présente l'ensemble des issues pour toutes les personnes détenues tout au long des procédures, selon qu'elles ont fait ou non l'objet d'un examen approfondi. 
Tableau 9

Issues des procédures pour les personnes détenues en cours de procédures selon la présence ou non d'examen approfondi

\begin{tabular}{|l|c|c|c|}
\hline \multirow{2}{*}{ ISSUE } & \multicolumn{3}{|c|}{ PRÉSENCE D'EXAMEN } \\
\cline { 2 - 4 } & $\begin{array}{c}\text { Détenu avec } \\
\text { examen }\end{array}$ & $\begin{array}{c}\text { Détenu sans } \\
\text { examen }\end{array}$ & Total \\
\hline \multirow{3}{*}{ Détention } & 4 & 7 & 11 \\
& 36,4 & 63,6 & 35,5 \\
\hline \multirow{2}{*}{ Sentence } & 18,2 & 77,7 & 5 \\
\hline \multirow{3}{*}{ OLspendue } & 3 & 2 & 16,1 \\
& 60,0 & 40,0 & 12 \\
\hline \multirow{3}{*}{ Libéré } & 13,6 & 22,2 & 38,7 \\
& 12 & & 3 \\
\hline Total & 100,0 & & 9,7 \\
\hline
\end{tabular}

Malgré le petit nombre d'effectifs, la distinction entre les deux groupes est très marquée. Pour les personnes ayant fait l'objet d'un examen et qui sont détenues, l'issue privilégiée est constituée par la voie psychiatrique; quant aux autres, la détention en cours de procédure a de fortes chances de se traduire au niveau de la sentence par la continuation de cette détention. Cette dimension psychiatrique se retrouve aussi parmi les conditions des personnes qui avaient été libérées en cours de procédure. En effet, pour 35 justiciables, le tribunal assortit la sentence de conditions diverses à caractère psychosocial ou psychiatrique. Ces conditions peuvent prendre plusieurs formes : suivi psychiatrique, prise de médicaments, cure de désintoxication... Bien que les problèmes potentiels de santé mentale des justiciables aient été réglés d'un point de vue procédural, le tribunal ne les perd pas de vue au moment du prononcé de la sentence.

\section{CONCLUSION}

Comme nous l'avions indiqué au début de notre étude, nous ne prétendons pas avoir procédé à une évaluation de l'application des nouvelles dispositions du Code criminel concernant les troubles mentaux. Néanmoins, les données dont nous disposons nous ont permis de constater que 
les pratiques du tribunal changent lentement, et que les nouvelles dispositions sont encore peu utilisées. Ces nouvelles dispositions, tout comme les anciennes d'ailleurs, ne sont pas sans rapport avec l'usage de la détention provisoire en cours de procédure. De tels liens doivent être lus à deux niveaux différents. D'abord, dans les dispositions législatives elles-mêmes. Le bref survol des modifications du Code criminel nous a permis de constater que cette question est centrale et qu'elle est encadrée de diverses façons. Soulignons ici la priorité de remise en liberté, clairement énoncée dans le texte, et qui devrait orienter les décisions du tribunal. Mais le texte ne se limite pas à cet énoncé de principe, puisqu'il prévoit des balises quant à la durée possible des différents types de procédures entraînant ou nécessitant une mise en détention des justiciables.

Mais c'est dans l'examen des décisions du tribunal que le lien entre détention provisoire et problèmes de santé mentale est le plus visible. On constate d'abord l'association quasi parfaite entre mise sous garde et renvoi pour examen approfondi afin de déterminer l'aptitude à comparaître. C'est donc dire que malgré l'orientation en faveur d'une remise en liberté que l'on retrouve dans le Code criminel, le tribunal continue de détenir afin de procéder à l'évaluation de l'aptitude. Par ailleurs, une fois la question de l'aptitude tranchée, un nombre important de justiciables sont remis en liberté, alors que les autres seront détenus jusqu'à la fin des procédures. Cette décision est, quant à elle, associée à l'issue des procédures et au type de sentence imposé par la cour. Le tribunal garde d'ailleurs à l'esprit les problèmes supputés de santé mentale des justiciables, puisque dans bon nombre de cas, ces décisions sont assorties de conditions à caractère thérapeutique. 


\section{RÉFÉRENCES}

ARBOLEDA-FLOREZ, J., \& HOLLEY, H. L. (1988), Criminalization of the Mentally IIl : Part II, Initial Detention, Canadian Journal of Psychiatry, 33, (mars) pp. 8795 .

CALGARY WHO Centre for Research and Training in Mental Health (1993), Bill C-30: The Alberta Experience.

CANADA, MINISTÈRE DU SECRÉTARIAT D'ÉTAT (1987), La charre canadienne des droits et libertés, Ottawa : Secrétariat d'État du Canada.

DAVIS, S. (1992), Assessing the "Criminalization » of the Mentally Ill in Canada, Canadian Journal of Psychiatry, 37 (8), pp. 532-538.

DAVIS, S. (1994), Fitness to Stand Trial in Canada in Light of the Recent Criminal Code Amendments, International Journal of Law and Psychiatry, 17 (3), pp. 319 329.

DESROSIERS, C. (Avocat). (s. d.), Le cautionnement et les garanties constitutionnelles. s.l., s.n.

HOLLEY, H. L., \& ARBOLEDA-FLOREZ, J. (1988), Criminalization of the Mentally Ill : Part I. Police Perceptions, Canadian Journal of Psychiatry, 33, pp. 81-86.

LABERGE, D., \& LANDREVILLE, P. (1993), Détention provisoire et remise en liberté avant jugement, L'état des recherches en Amérique du Nord, Revue internationale de criminologie et de police technique, XLVI (2), pp. 152-169.

LABERGE, D., \& MORIN, D. (1993), Troubles mentaux et intervention pénale ; questions entourant les êvaluations de la judiciarisation en Amérique du Nord, Déviance et Société, 17 (3), pp. 309-348.

LABERGE, D., \& LANDREVILLE. P., MORIN, D., ROBERT, M., \& SOULLIÈRE, N. (à paraître), Contrôle pénal et santé mentale, Montréal : PUM et DeBoeck.

LANDREVILLE, P. (1993), La prédiction de la dangerosité et la mise sous garde, Revue du Barreau, 52 (3), pp. 731-746.

MURPHY, G. R. (1986), Special Care: Improving the Police Response to the Mentally Disabled, Washington, D. C. : Police Executive Research Forum.

SCHELLENBERG, E. G., WASYLENKI,D., WEBSTER, C. D., \& GOERING, P. (1992), A Review of Arrests Among Psychiatric Patients, International Journal of Law and Psychiatry, 15 (3), pp. 25 )-264.

TEPLIN, L. A. (1983), The Criminalization of the Mentally Ill : Speculation in Search of Data, Psychological Bulletin, 94 (1), pp. 5467.

TEPLIN, L. A. (1984a), Criminalizing Mental Disorder : the Comparative Arrest Rate of the Mentally Ill, American Psychologist, 39 (7), pp. 794-803.

TEPLIN, L. A. (1984b), Managing Disorder, Police Handling of the Mentally IIl, in L. A. Teplin (ed.), Mental Health and Criminal Justice, (pp. 157-175), Beverly Hills : Sage.

TEPLIN, L. A., \& PRUETT, N. S. (1992), Police as Streetcomer Psychiatrist : Managing the Mentally III, International Journal of Law and Psychiatry, 15 (2), pp. 139-156. 